\title{
Alterações Neuropsicológicas na Doença Renal Crônica pré-dialítica e sua associação com o metabolismo do ferro
}

\author{
Neuropsychological Changes in Pre-dialysis Chronic Kidney \\ Disease and its association with iron metabolism
}

\section{Cambios neuropsicológicos en la enfermedad renal crónica previa a la diálisis y su asociación con el metabolismo del hierro}

Ana Laura Maciel Almeida1, Marilise de Andrade Paraizo², José Otávio do Amaral Correa ${ }^{3}$, Débora dos Santos Dias ${ }^{4}$, Renata Silva Almeida Abritta5, Lucas Fernandes Suassuna6, Leopoldo Antônio Pires7, Marcus Gomes Bastos ${ }^{8}$, Natália Maria da Silva Fernandes ${ }^{9}$

\begin{abstract}
1.Médica Neurologista, Doutora, Departamento de Clínica Médica, Faculdade de Medicina, Serviço de Neurologia da Universidade Federal de Juiz de Fora, Juiz de Fora-MG, Brasil.

2.Médica Neurologista, Mestre, Convidada do Departamento de Clínica Médica, Faculdade de Medicina, Serviço de Neurologia, Juiz de Fora-MG, Brasil.

3. Médico Patologista, Doutor, Faculdade de Farmácia e Bioquímica da Universidade Federal de Juiz de Fora, Juiz de Fora-MG, Brasil.

4.Farmacêutica, Mestre, Departamento de Farmácia e Bioquímica do Hospital Universitário da Universidade Federal de Juiz de Fora, Juiz de Fora-MG, Brasil.

5.Psicóloga, Profissional Liberal, Juiz de Fora-MG, Brasil.

6.Graduando na Faculdade de Medicina, Universidade Federal de Juiz de Fora, Juiz de Fora-MG, Brasil.

7.Médico Neurologista, Mestre, Departamento de Clínica Médica, Faculdade de Medicina, Serviço de Neurologia da Universidade Federal de Juiz de Fora, Juiz de Fora-MG, Brasil.

8. Médico Nefrologista, Doutor, Departamento de Clínica Médica, Faculdade de Medicina, Serviço de Nefrologia da Universidade Federal de Juiz de Fora, Juiz de Fora-MG, Brasil. Fundação Instituto Mineiro de Ensino e Pesquisa em Nefrologia (IMEPEN) e Núcleo Interdisciplinar de Ensino e Pesquisa em Nefrologia (NIEPEN) da Universidade Federal de Juiz de Fora.

9.Médica Nefrologista, Doutora, Departamento de Clínica Médica, Faculdade de Medicina, Serviço de Nefrologia da Universidade Federal de Juiz de Fora, Juiz de Fora-MG, Brasil. Fundação Instituto Mineiro de Ensino e Pesquisa em Nefrologia (IMEPEN) e Núcleo Interdisciplinar de Ensino e Pesquisa em Nefrologia (NIEPEN) da Universidade Federal de Juiz de Fora.
\end{abstract}

\section{Resumo}

Introdução. Comprometimento cognitivo (CC) e alterações neuropsicológicas diversas são comuns no paciente com doença renal crônica (DRC), porém pouco avaliadas. A anemia e a deficiência de ferro podem contribuir para estas alterações. Objetivo. Avaliar CC e alterações neuropsicológicas consideradas frequentes na DRC pré-dialítica e sua associação com alterações no metabolismo do ferro. Método. Estudo transversal com 54 pacientes entre 21 e 65 anos avaliados sociodemografica, clínica e laboratorialmente. Realizada triagem cognitiva completa, teste de rastreio de cognição global: Montreal Cognitive Assessment (MoCA), bateria de testes de memória, atenção, velocidade de processamento, fluência verbal e funções executivas, escalas de sono (Escala de Sonolência Diurna de Epworth, Questionário Clínico de Apneia Obstrutiva do Sono de Berlin (AOS), Questionário de cinco perguntas de sintomas de Pernas Inquietas, depressão (Inventário de depressão de Beck, Mini-Plus para Episódio Depressivo Maior (DSM-IV) e de funcionalidade (Questionário de Atividades Funcionais de Pfeffer). Resultados. Sintomas de AOS estiveram presentes em 76,9\%, pernas inquietas e sonolência diurna $(35,2 \%)$ e sintomas depressivos $(34,7 \%)$. O MoCA esteve alterado em $59,3 \%$. Correlação de Pearson entre testes neuropsicológicos e Hemoglobina(Hb), índice de Saturação de Transferrina(IST), ferritina e proteína C reativa utrassensível (PCRus), mostrou associação entre MoCA e $\mathrm{Hb}(r=0,310$ e $p=0,02)$. Regressão Linear utilizando três modelos ajustados por variáveis sociodemográficas e IST ou ferritina ou $\mathrm{Hb}$ : modelo 1 e 2-escolaridade e depressão se associaram ao MoCA, modelo 3-Hb e depressão se associaram ao MoCA. 
Conclusão. O nível sérico de $\mathrm{Hb}$ e a presença de depressão foram associadas com CC. Níveis mais baixos de ferritina se correlacionaram com o MoCA, enquanto não encontramos associação com os demais marcadores do metabolismo do ferro.

Unitermos. comprometimento cognitivo; cognição; depressão; doença renal crônica; deficiência de ferro

\begin{abstract}
Introduction. Cognitive impairment (CI) and diverse neuropsychological alterations are common in chronic kidney disease, but little evaluated. Anemia and iron deficiency can contribute to CC. Objective. to evaluate CI and the neuropsychological alterations considered frequent in pre-dialysis CKD its association with changes in iron metabolism. Method. Crosssectional study with 54 patients between 21 and 65 years old evaluated sociodemographically, clinically and laboratory. Complete cognitive screening, global cognition screening test: Montreal Cognitive Assessment (MoCA), battery of memory tests, attention, processing speed, verbal fluency and executive functions, sleep scales (Epworth Daytime Sleepiness Scale, Clinical Questionnaire Berlin Obstructive Sleep Apnea (OSA), Five Questions Questionnaire for Restless Legs Symptoms, Depression (Beck Depression Inventory, Mini-Plus for Major Depressive Episode (DSM-IV) and Functionality (Functional Activity Questionnaire Results. OSA symptoms were present in $76.9 \%$, restless legs and daytime sleepiness (35.2\%) and depressive symptoms (34.7\%). MoCA was altered in 59.3\%. Pearson correlation between tests neuropsychological and Hemoglobin ( $\mathrm{Hb})$, Transferrin Saturation Index (TSI), ferritin and Ultra-Sensitive C-Reactive Protein (US-CRP), showed an association between MoCA and $\mathrm{Hb}$ $(r=0.310, p=0.02)$. Linear regression using three models adjusted for sociodemographic and TSI or ferritin or $\mathrm{Hb}$ : model 1 and 2-education and depression were associated with MoCA, model $3-\mathrm{Hb}$ and depression were associated with MoCA. Conclusion. The serum level of $\mathrm{Hb}$ and the presence of depression were associated with CI. Lower levels of ferritin correlated with MoCA, while we found no association with other markers of iron metabolism.
\end{abstract}

Keywords. cognitive impairment; cognition; depression; chronic kidney disease; iron deficiency

\begin{abstract}
Resumen
Introducción. El deterioro cognitivo (CC) y diversos cambios neuropsicológicos son comunes en pacientes con enfermedad renal crónica (ERC), pero poco evaluados. La anemia y la deficiencia de hierro pueden contribuir a estos cambios. Objetivo. Evaluar la CC y los cambios neuropsicológicos considerados frecuentes en la ERC prediálisis y su asociación con cambios en el metabolismo del hierro. Método: Estudio transversal con 54 pacientes entre 21 y 65 años evaluados sociodemográficamente, clínicamente y en laboratorio. Examen cognitivo completo, prueba de detección cognitiva global: Montreal Cognitive Assessment (MoCA), batería de pruebas de memoria, atención, velocidad de procesamiento, fluidez verbal y funciones ejecutivas, escalas de sueño (Epworth Daytime Sleepiness Scale, Clinical Questionnaire Apnea obstructiva del sueño de Berlín (AOS), cuestionario de cinco preguntas para síntomas de piernas inquietas, depresión (inventario de depresión de Beck, Mini-Plus para episodio depresivo mayor (DSM-IV) y funcionalidad (cuestionario de actividad funcional) Resultados. Síntomas de AOS estuvo presente en $76.9 \%$, piernas inquietas y somnolencia diurna (35.2\%) y síntomas depresivos (34.7\%). MoCA se alteró en $59.3 \%$. Correlación de Pearson entre pruebas neuropsicológicos y de hemoglobina $(\mathrm{Hb})$, índice de saturación de transferrina (IST), ferritina y proteína $C$ reactiva ultrasensible (PCRus), mostraron una asociación entre MoCA y $\mathrm{Hb}(r=0.310, p=0.02)$. Regresión lineal utilizando tres modelos ajustados por variables sociodemográficas e IST o ferritina o $\mathrm{Hb}$ : los modelos 1 y 2 de educación y depresión se asociaron con MoCA, el modelo 3-Hb y la depresión se asociaron con MoCA. Conclusión. el nivel sérico de $\mathrm{Hb}$ y la presencia de depresión se asociaron con DC. Los niveles más bajos de ferritina se correlacionaron con MoCA, mientras que no encontramos asociación con otros marcadores del metabolismo del hierro.
\end{abstract}

Palabras clave. deterioro cognitivo; cognición; depresión; enfermedad renal crónica; deficiencia de hierro

Trabalho na Fundação Instituto Mineiro de Ensino e Pesquisa em Nefrologia (IMEPEN) e Núcleo Interdisciplinar de Ensino e Pesquisa em Nefrologia (NIEPEN) da Universidade Federal de Juiz de Fora, Juiz de Fora-MG, Brasil.

Conflito de interesse: não

Recebido em: 22/07/2020

Aceito em: 29/09/2020

Endereço para correspondência: Natália MS Fernandes, R. Jamil Altaf 132. Vale do Ipê. Juiz de ForaMG. CEP 36035-380. Tel: +55 32 88696319. E-mail: nataliafernandes02@gmail.com 


\section{INTRODUÇÃO}

De acordo com a Organização Mundial de Saúde existem no mundo cerca de 50 milhões de pessoas com demência, 60\% residem em países de baixa e média renda. A cada ano há 10 milhões de novos casos. A doença de Alzheimer é a forma mais comum de demência podendo ser responsável por 60-70\% dos casos $^{1}$.

Precedendo o diagnóstico de demência, os pacientes apresentam um comprometimento cognitivo inicialmente leve, frequentemente não diagnosticado e progressivo. Comprometimento cognitivo leve (CCL) é definido como uma alteração cognitiva que não altera a atividade de vida diária².

Outra importante patologia de importância epidemiológica internacional é a doença renal crônica (DRC) que, nos últimos 18 anos, apresentou um aumento de $212 \%$ do número de pacientes em diálise no Brasil ${ }^{3}$. Conceituamos doença renal crônica naquele indivíduo com diminuição na taxa de filtração glomerular estimada (TFGe) abaixo de 60 $\mathrm{ml} / \mathrm{min} / 1,73 \mathrm{~m}^{2}$ (com ou sem lesão estrutural) ou lesão do parênquima renal, baseado na presença de proteinúria ( $>30 \mathrm{mg} / \mathrm{dL}$ em urina isolada) ou hematúria, em pelo menos duas medidas e persistente por mais de três meses ${ }^{4}$.

Recente revisão confirma que pacientes com DRC correm um risco aumentado tanto de demência quanto de seu pródromo, o CCL, quando comparada a população geral e é caracterizado por déficits nas funções executivas, memória e atenção. Infelizmente, as intervenções atuais que visam fatores de risco cardiovascular (como anti- 
hipertensivos, agentes antiplaquetários e estatinas) parecem ter pouco ou nenhum efeito sobre o CCL associada à DRC, sugerindo que o acúmulo de neurotoxinas em pacientes urêmicos pode ser mais importante, além do fato de esta alteração ser multifatorial e ocorrer numa faixa etária mais jovem que na população geral, sendo pouco reconhecido, principalmente na fase inicial ${ }^{5,6}$. No contexto da demência e da DRC, a depressão apresenta suma importância por ser pródromo, diagnóstico diferencial e até consequência da primeira e por ter sua prevalência aumentada na segunda, quando comparada a população geral?.

Outras alterações neuropsicológicas como a apneia obstrutiva do sono ${ }^{8}$ e as síndromes das pernas inquietas ${ }^{9}$ são frequentemente associadas a DRC tanto dialítica quanto prédialítica, ambas têm sua fisiopatologia, neste contexto, multifatorial e ainda não completamente esclarecida.

A anemia é comum nos pacientes com doença renal, as causas mais frequentes são a deficiência de produção de eritropoietina e deficiência de ferro ${ }^{10}$. A prevalência de anemia varia de $9 \%$ a 64\% dependendo do estágio da DRC e da definição de anemia, e a deficiência de ferro atinge 30\% da população geral mundial sendo a causa mais importante de anemia. A deficiência de ferro pode levar a comprometimento cognitivo, mesmo sem anemia evidente e sem doença renal associada, independente da idade ${ }^{11}$.

A relevância do presente estudo está em avaliar de forma ampla, alterações neuropsicológicas frequentes, porém, nem sempre diagnosticadas no contexto da DRC e, 
portanto, não abordadas de forma precoce. Além disso, correlacioná-las com alterações clínicas muito comuns e reversíveis, observadas na DRC, que são as alterações associadas ao metabolismo do ferro com ou sem anemia para que tenhamos oportunidade de intervir, podendo, se possível modificar o curso de algumas destas alterações. 0 objetivo deste estudo foi avaliar alterações neuropsicológicas frequentes, comprometimento cognitivo e sua associação com alterações no metabolismo do ferro na DRC pré-dialítica.

\section{MÉTODO}

\section{Amostra}

Estudo transversal em amostra por conveniência. Todos os participantes assinaram o Termo de Consentimento Livre e Esclarecido (TCLE), aprovado pelo Comitê de Ética em Pesquisa da Plataforma Brasil sob o número 01995112.6.0000.5147.

Foram avaliados 54 pacientes nos ambulatórios do Centro Hiperdia da Fundação Instituto Mineiro de Ensino e Pesquisas em Nefrologia (IMEPEN) - Núcleo Interdisciplinar de Estudos e Pesquisa em Nefrologia (NIEPEN) Universidade Federal de Juiz de Fora (UFJF). Os dados foram coletados no período de abril de 2013 a dezembro de 2014 . Critérios de Inclusão: pacientes de 21 a 65 anos, independente de cor, sexo ou etnia, com DRC nos estágios iniciais e 5 não dialítico. Não foram incluídos pacientes com diagnóstico de psicoses, traumatismo cranioencefálico que 
tenha resultado em internação, comprometimento motor incapacitante, distúrbios visuais e auditivos não corrigidos e diagnóstico clínico de delirium. Foram avaliados dados sociodemográficos (idade, sexo, escolaridade, estado civil, causa da insuficiência renal, estágio da doença, comorbidades, história de alcoolismo, uso de medicações (benzodiazepínicos, opiáceos, sedativos e antidepressivos) e uso de estimulantes da eritropoese (eritropoetina - dose em uso). Os dados laboratoriais (em data próxima da avaliação cognitiva, até 3 meses) coletados foram: Hemoglobina $(\mathrm{g} / \mathrm{dL})$, sódio $(\mathrm{mEq} / \mathrm{L})$, potássio $(\mathrm{mEq} / \mathrm{L})$, glicemia de jejum $(\mathrm{mg} / \mathrm{dL})$, hormônio estimulante da tireóide $(\mathrm{TSH})(\mathrm{mcU} / \mathrm{mL})$, Ácido fólico(ng/mL), Vitamina B12 (pg/mL), VDRL, Ferritina sérica $(\mathrm{ng} / \mathrm{mL})$, Uréia $(\mathrm{mg} / \mathrm{dL})$, Creatinina $(\mathrm{mg} / \mathrm{dL})$, Cálcio $(\mathrm{mg} / \mathrm{dL})$, Magnésio $(\mathrm{mg} / \mathrm{dL})$, Albumina $(\mathrm{g} / \mathrm{dL})$, Alanina aminotransferase (ALT) (U/L), Aspartato aminotransferase $(A S T)(U / L)$, Colesterol total $(\mathrm{mg} / \mathrm{dL}), \mathrm{HDL}(\mathrm{mg} / \mathrm{dL})$, Triglicérides $(\mathrm{mg} / \mathrm{dL})$, Paratormônio Intacto (PTHi) $(\mathrm{pg} / \mathrm{mL})$, Ferro sérico $(\mathrm{mcg} / \mathrm{dL})$, Índice de saturação da transferrina (IST)(\%), Proteína C Reativa ultra sensível (PCRus) (mcg/L) e 25-hidroxi-vitamina $\mathrm{D}(\mathrm{ng} / \mathrm{mL})$.

\section{Avaliação Neuropsicológica}

Realizada triagem cognitiva com o paciente e uma entrevista com um acompanhante do paciente sobre atividades funcionais. Quando o paciente comparecia sozinho, essa entrevista com o acompanhante era realizada por telefone. Na avaliação cognitiva foram utilizados um 
teste de rastreio cognitivo global: Montreal Cognitive Assessment (MoCA $)^{12}$ e bateria de testes de memória, atenção, velocidade de processamento, fluência verbal e funções executivas ${ }^{13-18}$. Além destes, foram avaliadas outras alterações neurológicas pelos seguintes instrumentos: um questionário de atividades funcionais e duas escalas de depressão. Observamos no quadro 1 o Questionário de Atividades Funcionais de Pfeffer ${ }^{19}$, Escala de Sonolência Diurna de Epworth ${ }^{20}$, Questionário Clínico de Apneia Obstrutiva do Sono de Berlin (AOS), Questionário de cinco perguntas de sintomas de Pernas Inquietas (SPI) ${ }^{21}$ (Consenso Pernas Inquietas, 2015), Inventário de depressão de Beck $(B D I)^{22}$, Mini-Plus para Episódio Depressivo Maior $(\mathrm{DSM}-\mathrm{IV})^{23}$.

O tempo médio de duração da avaliação foi de aproximadamente 60 minutos.

\section{Análise Estatística}

Inicialmente foi realizada uma análise descritiva dos dados que foram expressos como média, desvio padrão, mediana, percentagem ou frequência conforme a característica da variável. Os pacientes foram separados em dois grupos conforme o nível do IST e também da ferritina sérica (baseados em valores considerados normais para pacientes com DRC). Para comparar as variáveis sociodemográficas, clínicas e laboratoriais entre pacientes com IST $>$ e $<20 \%$ e Ferritina $>$ e $<100$ utilizamos o teste $\mathrm{t}$ de Student para amostras independentes, Mann Whitney 
ou Qui-Quadrado. Realizado teste de Correlação de Pearson ou Spearman (conforme a característica da variável) entre Hemoglobina, IST, Ferritina e PCRus e testes neuropsicológicos. Devido ao pequeno número de pacientes por categoria da DRC esta variável foi tratada de forma contínua e usado a TFGe no teste de correlação e nos modelos de regressão. Finalmente, foi realizada uma Regressão Linear tendo como variável desfecho o MoCA e variáveis independentes: idade, escolaridade, diabetes mellitus, depressão, TFGe e no modelo 1: associada a variável IST; modelo 2: Ferritina e no modelo 3: Hemoglobina. As variáveis relacionadas ao metabolismo do ferro não foram colocadas em um mesmo modelo pela colinearidade. Foi assumido $p \leq 0,05$, intervalo de confiança de $95 \%$, e utilizado o SPSS 17.0 .

\section{RESULTADOS}

Foram convidados 111 pacientes; 13 se recusaram a participar, 4 apresentaram problemas clínicos e 14 participaram do estudo piloto. Oitenta pacientes fizeram a avaliação neuropsicológica, e 26 não realizaram exames laboratoriais no período da avaliação, totalizando 54 pacientes incluídos (Figura 1). 
Figura 1. Fluxograma da amostra do estudo.

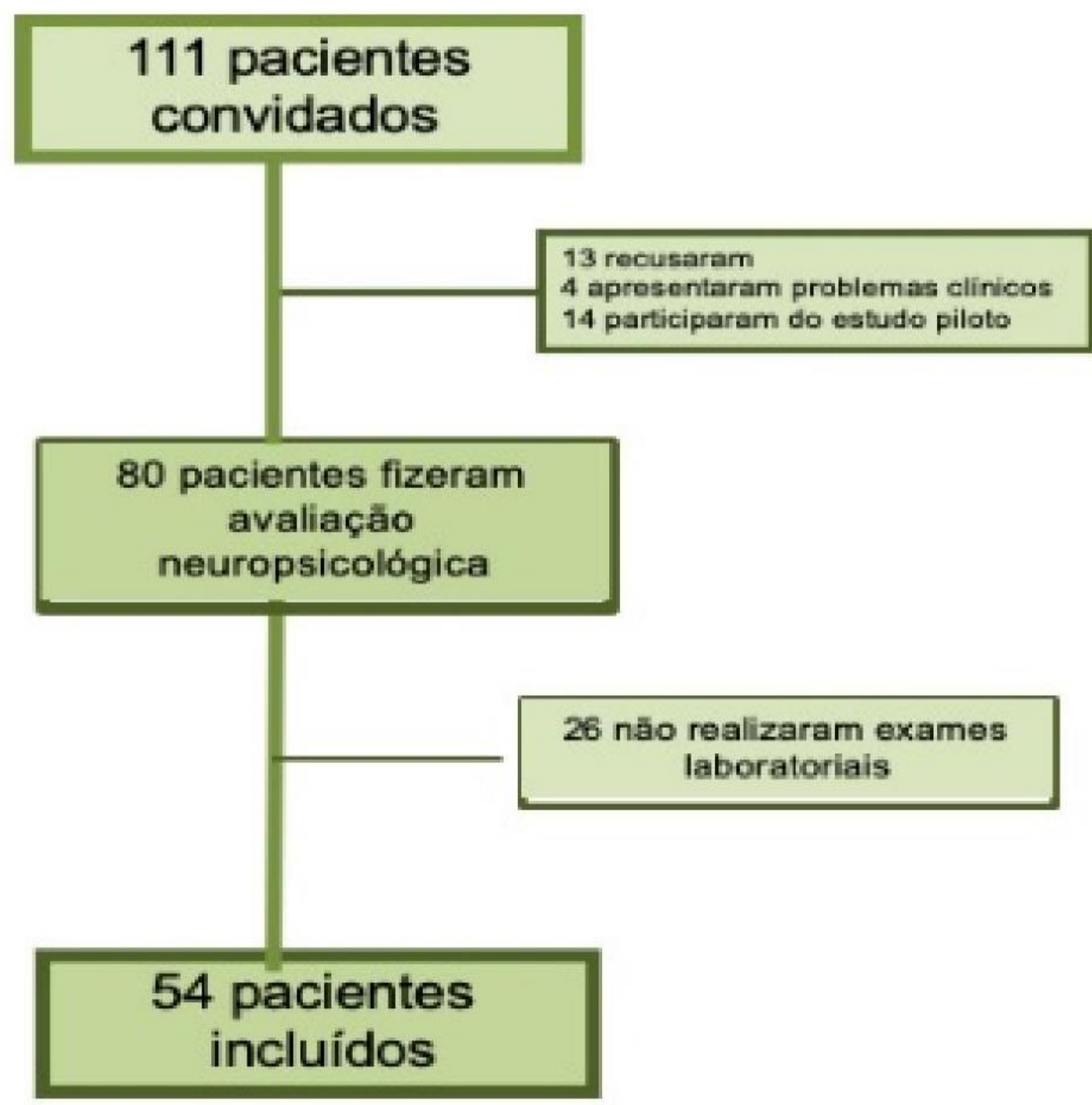

\section{Características Clínicas e Sociodemográficas}

As características sociodemográficas e clínicas dos participantes estão descritas na Tabela 1. Cinco pacientes eram anêmicos $(9,2 \%)$, sendo que dois pacientes estavam fazendo reposição de ferro (um paciente usando sulfato ferroso, uma paciente usando sacarato de hidróxido férrico (Noripurum ${ }^{\circledR}$ ) e uma paciente usando eritropoietina recombinante. Importante referir que os pacientes com hipotireoidismo estavam em tratamento e compensados. 
Tabela 1. Características sócio demográficas e clínicas dos participantes.

\begin{tabular}{|c|c|}
\hline Variáveis & Dados \\
\hline $\begin{array}{l}\text { Idade (anos) Média } \pm d p \\
\text { Mediana (p25-p75) }\end{array}$ & $\begin{array}{l}56,89 \pm 7,69 \\
58(54,75-62,25)\end{array}$ \\
\hline $\begin{array}{r}\text { Gênero (\%) Masculino }(n=31) \\
\text { Feminino }(n=23)\end{array}$ & $\begin{array}{l}57,4 \\
42,6\end{array}$ \\
\hline $\begin{array}{l}\text { Estado Civil }(\%) \\
\text { Casado ou união estável }(n=34) \\
\text { Solteiro }(n=3) \\
\text { Viúvo ou separado }(n=17)\end{array}$ & $\begin{array}{l}63 \\
5,6 \\
31,4\end{array}$ \\
\hline $\begin{array}{l}\text { Escolaridade }(\%) \text { (Dados Faltantes=2) } \\
\text { Anos de escolarização } \\
\leq 4 \text { anos }(n=28) \\
5-8 \text { anos }(n=15) \\
>8 \text { anos }(n=9)\end{array}$ & $\begin{array}{l}53,8 \\
28,9 \\
17,3\end{array}$ \\
\hline $\begin{array}{l}\text { Categoria da DRC (\%) } \\
1(n=4) \\
2(n=7) \\
3(n=23) \\
4(n=16) \\
5(n=4)\end{array}$ & $\begin{array}{l}7,4 \\
13 \\
42,6 \\
29,6 \\
7,4\end{array}$ \\
\hline Etilismo $(\%)(n=4)$ & 7,8 \\
\hline Tabagismo $(\%)(n=11)$ & 21,2 \\
\hline $\begin{array}{l}\text { Comorbidades }(\%) \\
\operatorname{HAS}(n=50) \\
\operatorname{DM}(n=26) \\
\operatorname{DCV}(n=14)\end{array}$ & $\begin{array}{l}92,6 \\
49,1 \\
31,8\end{array}$ \\
\hline Hipotireoidismo $(n=5)$ & 10,9 \\
\hline Medicamentos (\%) (Dados faltantes = & \\
\hline $\begin{array}{l}\text { Benzodiazepínico }(n=8) \\
\text { Antidepressivo }(n=9) \\
\text { Hipnótico }(n=1) \\
\text { Anticonvulsivante }(n=1) \\
\text { Estimuladores da Eritropoetina }(n=1) \\
\text { Ferro }(n=2) \\
\text { IECA }(n=22) \\
\text { Beta bloqueador }(n=26) \\
\text { Diurético }(n=41) \\
\text { Estatina }(n=23) \\
\text { Vitamina } D(n=13) \\
\text { Hipoglicemiante }(n=23) \\
\text { Hormônio tireoidiano }(n=3)\end{array}$ & $\begin{array}{l}16 \\
18 \\
2,1 \\
2,1 \\
2,1 \\
4,2 \\
45,8 \\
54,2 \\
85,4 \\
47,9 \\
27,1 \\
47,9 \\
6,3\end{array}$ \\
\hline
\end{tabular}

HAS = hipertensão arterial sistêmica, $\mathrm{DM}=$ diabetes mellitus, $\mathrm{DCV}=$ doença cardiovascular, $\mathrm{AOS}=$ apneia obstrutiva do sono, BDI= Inventário de Depressão de Beck, IECA= inibidor seletivo de enzima conversora de angiotensina. 
Os pacientes, em geral, estavam bem controlados laboratorialmente, sem alterações mais expressivas, conforme mostrado na Tabela 2 , porém apresentavam níveis elevados de PCRus $(8,00 \pm 15,96$, mediana 2,7$)$.

Tabela 2. Tabela com dados descritivos dos exames laboratoriais, média $\pm \mathrm{dp}$ (e/ou mediana (P25-P75) para os dados não normais).

\begin{tabular}{ll}
\hline Variável & Média \pm dp \\
\hline Creatinina $(\mathrm{mg} / \mathrm{dL})$ & $2,15 \pm 1,46$ \\
Ureia $(\mathrm{mg} / \mathrm{dL})$ & $64,62 \pm 37,75$ \\
Sódio $(\mathrm{mEq} / \mathrm{L})$ & $138,35 \pm 3,55$ \\
Potássio $(\mathrm{mEq} / \mathrm{L})$ & $4,54 \pm 0,66$ \\
Magnésio $(\mathrm{mg} / \mathrm{dL})$ & $1,82 \pm 0,26$ \\
Cálcio $(\mathrm{mg} / \mathrm{dL})$ & $9,49 \pm 0,78$ \\
PTHi $(\mathrm{pg} / \mathrm{mL})$ & $185,53 \pm 219,94$ \\
& Mediana $108,55(70,65-198,75)$ \\
PCRus $(\mathrm{mg} / \mathrm{L})$ & $8,00 \pm 15,96$ \\
& Mediana $2,7(0,98-7,98)$ \\
Glicose $(\mathrm{mg} / \mathrm{dL})$ & $119 \pm 47,93$ \\
IST $(\%)$ & $27,30 \pm 9,54$ \\
Ferritina $(\mathrm{ng} / \mathrm{mL})$ & $130,55 \pm 101,02$ \\
Ferro sérico $(\mu \mathrm{g} / \mathrm{dL})$ & $72,09 \pm 28,67$ \\
Hemoglobina $(\mathrm{g} / \mathrm{dL})$ & $13,23 \pm 1,86$ \\
Colesterol Total $(\mathrm{mg} / \mathrm{dL})$ & $170,38 \pm 43,50$ \\
HDL $(\mathrm{mg} / \mathrm{dL})$ & $40,59 \pm 12,14$ \\
Triglicérides $(\mathrm{mg} / \mathrm{dL})$ & $175,61 \pm 117,77$ \\
AST $(\mathrm{U} / \mathrm{L})$ & $23,98 \pm 13,70$ \\
ALT $(\mathrm{U} / \mathrm{L})$ & $18,83 \pm 14,37$ \\
Albumina $(\mathrm{g} / \mathrm{dL})$ & $4,04 \pm 0,39$ \\
TSH us $(\mu \mathrm{UI} / \mathrm{mL})$ & $2,82 \pm 3,16$ \\
& Mediana $2,01(1,36-3,45)$ \\
Vitamina B12 $(\mathrm{pg} / \mathrm{mL})$ & $382,65 \pm 217,28$ \\
Ácido Fólico $(\mathrm{ng} / \mathrm{mL})$ & $9,20 \pm 3,85$ \\
VDRL positivo $(\%)$ & $1,85(\mathrm{n}=1)$ \\
\hline
\end{tabular}

PTHi $=$ Paratormônio intacto, PCRus= Proteína C Reativa ultra sensível, IST = Índice de Saturação de Transferrina, AST = aspartato aminotransferase, ALT = alanina aminotransferase,TSHus= hormônio estimulante da tireóide ultra sensível.

\section{Avaliação Neuropsicológica}

$\mathrm{Na}$ avaliação neuropsicológica, o MoCA foi o teste de rastreio que mais evidenciou prejuízo cognitivo, estando alterado em $59,3 \%$ da amostra mesmo diminuindo o ponto de corte sugerido para população brasileira de $\leq 25$ pontos, para $\leq 24$ pontos, para ajustar à escolaridade da amostra ${ }^{17}$. 
Houve correlação entre o MoCA alterado e os anos de estudo ( $r$ de Pearson $=0,366, p=0,008 ;$ Tabela 3).

Tabela 3. Percentagem de alteração do MoCA $\leq 24$ pontos e anos de estudo (Teste do Qui Quadrado).

\begin{tabular}{ccc}
\hline Anos de Estudo/MoCA & Alterado (\%) & Normal (\%) \\
\hline$\leq 4$ & 58,5 & 36,4 \\
\hline $5-8$ & 29,3 & 27,3 \\
\hline$\geq 8$ & 12,2 & 36,3 \\
\hline
\end{tabular}

Correlação de Pearson entre o MoCA e anos de estudo: $r=0,366$ e $p=0,008$ (as variáveis neste teste foram usadas como numéricas contínuas)

Entre os pacientes avaliados, $34,7 \%$ apresentaram sintomas depressivos pelo BDI e 24,1\% tinham depressão pelo MiniPlus; desses, apenas 46,15\% estavam em tratamento medicamentoso (Tabelas 1 e 4 ).

Tabela 4. Questionários e escalas de avaliação neuropsicológica.

\begin{tabular}{lc}
\hline Questionários e Escalas (\%) & $(\%)$ \\
\hline Sonolência diurna (Epworth $>10)(n=19)$ & 35,2 \\
\hline Síndrome das pernas inquietas $(n=19)$ & 35,2 \\
\hline AOS *(Berlim alto risco) $(n=40)$ & 76,9 \\
\hline Depressão (MINI PLUS) $(n=13)$ & 24,1 \\
\hline BDI ( $\geq 13$ pontos) ( $=17)$ & 34,7 \\
\hline Perda de funcionalidade (Pfeffer $\geq 5)(n=6)$ & 12,2 \\
\hline
\end{tabular}

*Apneia Obstructive Scale **Beck Depression Inventary

O comprometimento da funcionalidade, avaliado pelo Questionário de Atividades Funcionais de Pfeffer, foi observado em $12,2 \%$ dos pacientes (Tabela 4 ). 
$\mathrm{Na}$ amostra avaliada, 76,9\% dos pacientes apresentaram alto risco para apneia obstrutiva do sono. Mais de $35 \%$ dos pacientes apresentaram sintomas clínicos de pernas inquietas e sonolência diurna. Dos 26 pacientes com diabetes mellitus (DM), $9(34,6 \%)$ apresentaram sintomas de SPI, e, dos 28 pacientes sem DM, $10(35,7 \%)$ apresentaram sintomas de SPI (Tabela 4).

Foram feitas comparações entre variáveis sociodemográficas e clínicas entre pacientes com IST $<20 \%$ e $>20 \%$ (Tabela 5), e com Ferritina $<100 \mathrm{ng} / \mathrm{mL}$ e $>100 \mathrm{ng} / \mathrm{mL}$ (Tabela 6), bem como comparações entre variáveis laboratoriais e esses índices, não sendo observadas alterações significativas nas variáveis sociodemográficas e clínicas. Entre as variáveis laboratoriais, os pacientes com IST $<20 \%$ apresentaram PCRus mais elevado (PCRus: 17,28 $\pm 27,38$ versus 5,12 $\pm 8,76, p=0,02$ ).

As Tabelas 5 e 6 descrevem as comparações dos testes neuropsicológicos utilizados entre pacientes com IST $<20 \%$ e>20\%, e os pacientes com ferritina $<100 \mathrm{ng} / \mathrm{mL}$ e $>100 \mathrm{ng} / \mathrm{mL}$ e respectivos valores de significância. Observamos que pacientes com níveis de ferritina mais baixos apresentaram MoCA alterado em maior percentagem (89,3\% versus $62,5 \%, p=0,02)$. 
Tabela 5. Comparação entre os Testes Neuropsicológicos entre pacientes com índice de saturação de transferrina (IST) $<20 \%$ e $>20 \%$ (Dados faltantes $=4$ )

\begin{tabular}{|c|c|c|c|}
\hline Variável & $\begin{array}{c}\text { IST }<20 \% \\
(n=11)\end{array}$ & $\begin{array}{l}\text { IST > 20\% } \\
(n=39)\end{array}$ & $\mathbf{p}$ \\
\hline Funcionalidade (Pfeffer $\geq 5$ ) & 0 & $16,2(n=6)$ & 0,07 \\
\hline Sintomas de Pernas inquietas (\%) & $27,3(n=3)$ & $38,5(n=15)$ & 0,48 \\
\hline AOS (Berlin) (\%) & $63,6(n=7)$ & $81,1(n=30)$ & 0,24 \\
\hline Sonolência Diurna (Epworth) (\%) & $45,5(n=5)$ & $30,8(n=12)$ & 0,37 \\
\hline Média $\pm d p$ & $8,64 \pm 5,87$ & $9,41 \pm 52$ & 0,69 \\
\hline Depressão (Miniplus) (\%) & $18,2(n=2)$ & $23,1(n=9)$ & 0,72 \\
\hline BDI $(\%)$ & $36,4(n=4)$ & $35,3(n=12)$ & 0,94 \\
\hline $\operatorname{MoCA}(\leq 24)(\%)$ & $90,9(n=10)$ & $74,4(n=29)$ & 0,20 \\
\hline \multicolumn{4}{|l|}{ TDR (média $\pm d p)$} \\
\hline Fluência Verbal Fonêmica & $4,09 \pm 1,13$ & $4,15 \pm 1,08$ & 0,86 \\
\hline $\mathrm{F}$ & $9,18 \pm 2,4$ & $9,87 \pm 3,99$ & 0,58 \\
\hline A & $6,64 \pm 1,62$ & $7,59 \pm 3,84$ & 0,42 \\
\hline $\mathrm{S}$ & $6,45 \pm 2,0$ & $7,97 \pm 3,26$ & 0,07 \\
\hline FAS & $22,27 \pm 4,69$ & $25,49 \pm 9,79$ & 0,13 \\
\hline Fluência Verbal Semântica (Animais) & $14,36 \pm 3,29$ & $14,08 \pm 4$ & 0,81 \\
\hline TEPIC-M & $10,45 \pm 2,97$ & $12,54 \pm 4,76$ & 0,08 \\
\hline Memória Lógica I & $18,55 \pm 6,60$ & $19,66 \pm 7,89$ & 0,66 \\
\hline Memória Lógica II & $15,18 \pm 8,75$ & $15,69 \pm 7,54$ & 0,84 \\
\hline Códigos & $27,64 \pm 12,16$ & $28,53 \pm 12,86$ & 0,83 \\
\hline Dígitos OD & $4,64 \pm 0,92$ & $4,72 \pm 0,97$ & 0,80 \\
\hline Dígitos OI & $3,09 \pm 0,83$ & $3,23 \pm 1,00$ & 0,64 \\
\hline \multicolumn{4}{|l|}{ Punho Borda Mão (\%) } \\
\hline Fez 3 vezes com examinador & 18,2 & 7,7 & \\
\hline Fez até 3 vezes sozinho & 9,1 & 20,5 & 0,46 \\
\hline Fez 6 vezes sozinho & 72,7 & 71,8 & \\
\hline \multicolumn{4}{|l|}{ Memória 10 figuras (média $\pm d p$ ) } \\
\hline Percepção visual & $10 \pm 0$ & $9,97 \pm 0,18$ & 0,32 \\
\hline Nomeação & $10 \pm 0$ & $9,97 \pm 0,18$ & 0,32 \\
\hline Memória incidental & $6,3 \pm 1,24$ & $5,62 \pm 1,01$ & 0,31 \\
\hline Memória Imediata I & $7,63 \pm 1,59$ & $7,76 \pm 1,12$ & 0,82 \\
\hline Memória Imediata II & $8,38 \pm 1,40$ & $8,59 \pm 1,08$ & 0,70 \\
\hline Evocação & $7,75 \pm 1,38$ & $7,69 \pm 1,36$ & 0,91 \\
\hline Reconhecimento & $9,38 \pm 0,74$ & $9,69 \pm 0,54$ & 0,29 \\
\hline
\end{tabular}

AOS = apneia obstrutiva do sono, BDI= Inventário de Depressão de Beck, MoCA= Montreal Cognitive Assessment, $\mathrm{TDR}=$ Teste do Desenho do Relógio, TEPIC= Teste Pictórico de Memória, Dígitos OD e OI= dígitos ordem direta e ordem inversa. 
Tabela 6. Comparação entre os Testes Neuropsicológicos entre Pacientes com Ferritina $<100 \mathrm{ng} / \mathrm{mL}$ e $>100 \mathrm{ng} / \mathrm{mL}$ (Dados faltantes $=2$ ).

\begin{tabular}{|c|c|c|c|}
\hline Variável & $\begin{array}{l}\text { Ferritina } \\
<100 \mathrm{ng} / \mathrm{mL} \\
(\mathrm{n}=28)\end{array}$ & $\begin{array}{l}\text { Ferritina } \\
>100 \mathrm{ng} / \mathrm{mL} \\
(\mathrm{n}=24)\end{array}$ & $\mathbf{p}$ \\
\hline Funcionalidade (Pfeffer $\geq 5$ ) $(\%)$ & $8,7(n=2)$ & $16,7(n=4)$ & 0,40 \\
\hline Média $\pm \mathrm{dp}$ & $0,7 \pm 1,8$ & $1,8 \pm 2,2$ & 0,07 \\
\hline Sintomas de Pernas inquietas (\%) & $39,3(n=11)$ & $33,3(n=8)$ & 0,65 \\
\hline Média $\pm d p$ & $1,89 \pm 2$ & $1,75 \pm 2,13$ & 0,80 \\
\hline AOS (Berlin) (\%) & $81,55(n=22)$ & $73,9(n=17)$ & 0,52 \\
\hline Sonolência Diurna (Epworth) (\%) & $42,9(n=12)$ & $29,2(n=7)$ & 0,30 \\
\hline Média $\pm d p$ & $9,54 \pm 5,29$ & $9 \pm 5,24$ & 0,71 \\
\hline Depressã̃o (Miniplus) (\%) & $25(n=7)$ & $25(n=6)$ & 0,62 \\
\hline $\mathrm{BDI}(\%)$ & $37,55(n=9)$ & $34,8(n=8)$ & 0,54 \\
\hline Média \pm dp & $10,72 \pm 9,59$ & $14,46 \pm 14,06$ & 0,28 \\
\hline $\operatorname{MoCA}(\leq 24)(\%)$ & $89,3(n=25)$ & $62,5(n=15)$ & 0,02 \\
\hline Média $\pm d p$ & $21,14 \pm 3,68$ & $22,71 \pm 4,22$ & 0,16 \\
\hline TDR $(\%)$ & $14,3(n=4)$ & $8,3(n=2)$ & 0,49 \\
\hline Média $\pm \mathrm{dp}$ & $4,14 \pm 1,04$ & $4,25 \pm 1,03$ & 0,71 \\
\hline \multicolumn{4}{|l|}{ Fluência Verbal Fonêmica } \\
\hline Média $\pm d p$ & $9,18 \pm 3,17$ & $10,46 \pm 4,04$ & 0,20 \\
\hline $\mathrm{F}$ & $7,04 \pm 3,46$ & $7,79 \pm 3,71$ & 0,45 \\
\hline A & $7,25 \pm 3,19$ & $8,25 \pm 3,3$ & 0,23 \\
\hline \multirow{2}{*}{\multicolumn{4}{|c|}{ FAS }} \\
\hline & & & \\
\hline Semântica & & & \\
\hline (Animais) (Média $\pm d p)$ & $14,11 \pm 4,58$ & $14,54 \pm 3,43$ & 0,70 \\
\hline TEPIC-M (Média $\pm d p)$ & $12,5 \pm 5,48$ & $12,38 \pm 4,74$ & 0,93 \\
\hline Memória Lógica I (Média士dp) & $18,04 \pm 7,86$ & $22,0 \pm 7,43$ & 0,06 \\
\hline Memória Lógica II (Média $\pm d p)$ & $15,11 \pm 8,74$ & $17,5 \pm 7,27$ & 0,29 \\
\hline Códigos (Média $\pm \mathrm{dp}$ ) & $27,5 \pm 14,8$ & $32,65 \pm 13$ & 0,20 \\
\hline Dígitos OD (\%) & $50(n=14)$ & $66,7(n=16)$ & \\
\hline Média $\pm \mathrm{dp}$ & $4,57 \pm 0,92$ & $4,6 \pm 0,95$ & 0,22 \\
\hline Dígitos OI (\%) & $67,9(n=19)$ & $91,7(n=22)$ & \\
\hline Média $\pm \mathrm{dp}$ & $3,21 \pm 1,03$ & $3,33 \pm 1$ & 0,03 \\
\hline \multicolumn{4}{|l|}{ Punho Borda Mão (\%) } \\
\hline Fez 3 vezes com examinador & $10,7(n=3)$ & $8,3(n=2)$ & \\
\hline Fez até 3 vezes sozinho & $14,3(n=4)$ & $20,8(n=5)$ & 0,92 \\
\hline \multirow{2}{*}{\multicolumn{4}{|c|}{ Memória 10 figuras (Média $\pm \mathrm{dp}$ ) }} \\
\hline & $10 \pm 0$ & $9,95 \pm 0,22$ & 0,31 \\
\hline Nomeação & $10 \pm 0$ & $9,95 \pm 0,22$ & 0,31 \\
\hline Memória incidental & $6,15 \pm 1,04$ & $5,42 \pm 0,96$ & 0,02 \\
\hline Memória Imediata I & $7,85 \pm 1,30$ & $7,68 \pm 1,10$ & 0,67 \\
\hline Memória Imediata II & $8,75 \pm 1,16$ & $8,47 \pm 1,17$ & 0,46 \\
\hline Evocação & $8 \pm 1,48$ & $7,68 \pm 1,37$ & 0,49 \\
\hline Reconhecimento & $9,55 \pm 0,68$ & $9,78 \pm 0,45$ & 0,32 \\
\hline
\end{tabular}

Realizada correlação de Pearson entre testes neuropsicológicos e Hemoglobina, IST, ferritina e PCRus, sendo que, dos testes avaliados, houve correlação apenas do 
MoCA com a hemoglobina $(r=0,310$ e $p=0,02)$ (dados não mostrados).

Tabela 7. Regressão linear tendo como variável dependente o MoCa e variáveis independentes: Idade, escolaridade, diabetes mellitus (DM), depressão (Mini-Plus), taxa de filtração glomerular estimada (TFGe), índice de saturação de transferrina (IST) ou Ferritina ou Hemoglobina.

\begin{tabular}{llll}
\hline $\begin{array}{l}\text { MODELO 1: } \\
\text { Variável }\end{array}$ & $\begin{array}{l}\text { IST } \\
\text { Coeficiente } \boldsymbol{\beta}\end{array}$ & IC 95\% & $\mathbf{p}$ \\
\hline Idade & 0,028 & $-0,13$ a 0,19 & 0,73 \\
Escolaridade & 1,66 & 0,12 a 3,19 & 0,03 \\
DM (Sim) & $-0,511$ & $-2,71$ a 1,69 & 0,64 \\
Depressão (Sim) & $-4,55$ & $-7,13$ a $-1,97$ & 0,001 \\
TFGe & $-0,06$ & $-0,05$ a 0,03 & 0,78 \\
IST & 0,099 & $-0,14$ a 0,21 & 0,08 \\
\hline MODELO 2: & Ferritina & IC 95\% & $\mathbf{p}$ \\
Variável & Coeficiente $\boldsymbol{\beta}$ & $-0,196$ a 0,150 & 0,78 \\
\hline Idade & $-0,023$ & 0,355 a 3,514 & 0,018 \\
Escolaridade & 1,93 & $-1,90$ a 2,07 & 0,93 \\
DM (Sim) & 0,08 & $-6,05$ a $-0,666$ & 0,016 \\
Depressão (Sim) & $-3,36$ & $-0,057$ a 0,037 & 0,67 \\
TFGe & $-0,01$ & $-0,013$ a 0,011 & 0,86 \\
Ferritina & $-0,001$ & & \\
\hline MODELO 3: & Hemoglobina & IC 95\% & $\mathbf{p}$ \\
Variável & Coeficiente $\boldsymbol{\beta}$ & $-0,20$ a 0,09 & 0,47 \\
\hline Idade & $-0,055$ & $-0,173$ a 2,732 & 0,08 \\
Escolaridade & 1,28 & $-1,32$ a 2,15 & 0,63 \\
DM & 0,418 & $-5,68$ a $-0,74$ & 0,01 \\
Depressão & $-3,21$ & $-0,101$ a 0,004 & 0,06 \\
TFGe & $-0,049$ & 0,162 a 1,516 & 0,016 \\
Hb & 0,839 & & \\
\hline MoCA= Montreal Cognitive Assessment, DM= diabetes mellitus, TFGe $=$ & taxa de filtração glomerular \\
estimada, IST= índice de saturação de transferrina, Hb= hemoglobina. &
\end{tabular}

A Regressão Linear, mostrada na Tabela 7, foi feita tendo como variável dependente o MoCA e variáveis independentes: idade, escolaridade, DM, depressão (Mini Plus) e TFGe, utilizando três modelos: no modelo 1 acrescentamos IST, no modelo 2 a ferritina e no modelo 3 a hemoglobina. No modelo 1 e 2, o maior nível de escolaridade associou-se a maiores escores do MoCA. Também nestes modelos maiores escores de depressão foram associadas a menores escores do MoCA. Porém, no modelo 3, apesar da depressão manter a mesma relação com o MoCA, níveis mais 
elevados de hemoglobina foram associados a maiores escores do MoCA.

\section{DISCUSSÃO}

A deficiência de ferro é causa frequente de anemia na DRC e está associada com comprometimento cognitivo ${ }^{24}$, porém na população estudada, encontramos associação independente da hemoglobina com cognição avaliada pelo MoCA.

A nossa amostra se caracterizou por ser jovem $(56,89 \pm 7,69)$, pois foram excluídos pacientes com mais de 65 anos para diminuir a possibilidade de comprometimento cognitivo relacionado a doenças degenerativas associadas à idade. Havia 53\% de pacientes com menos de 4 anos de estudo, porém os testes foram corrigidos pela escolaridade. Este dado mostra uma maior prevalência do que os dados do INAF de 2018 que revelam um percentual de $29 \%$ de analfabetismo funcional conquanto utilize testes padronizados para este fim e não apenas número de anos de estudo ${ }^{25}$.

Houve alta prevalência de comorbidades como hipertensão arterial sistêmica (HAS) $(92,6 \%)$, DM $(49,1 \%)$ e doenças cardiovasculares (31,8\%), em concordância com a literatura6.

Laboratorialmente, observamos que os pacientes se apresentam inflamados conforme demonstra a literatura, avaliados pela PCRus ${ }^{26}$. 
Como rastreio cognitivo, utilizamos o MoCA porque, apesar de ter sido validado no Brasil para uma população mais idosa, seu uso, em nosso estudo, se justifica, visto que a DRC e suas comorbidades colocam o paciente numa condição de envelhecimento precoce e fragilidade como visto nos idosos. O maior percentual de pacientes com MoCA alterado esteve no grupo com escolaridade menor que 4 anos. Sugere-se que este teste seja utilizado para indivíduos com escolaridade acima de 4 anos. Porém, acreditamos que, como no Brasil, os anos de estudo não predizem a capacidade de leitura e escrita, o indivíduo pode ser alfabetizado funcional sem ter frequentado escola e analfabeto funcional tendo mais de quatro anos de escolaridade, segundo dados do $\operatorname{INAF}^{25}$, este fato não invalida os achados. Outra informação relevante é que o IBGE $^{27}$ considera alfabetizado o indivíduo que consegue ler e escrever um bilhete simples na língua de origem. A realidade da nossa população é de pacientes com baixa escolaridade com diferentes graus de analfabetismo funcional independente dos anos de estudo, portanto é interessante avaliar os pacientes em situações clínicas reais.

A depressão/sintomas depressivos avaliada pelo (MINI PLUS) e pelo BDI também apresentou alta prevalência como vimos no estudo de Alsaleh et al. Como no estudo descrito, a prevalência de pacientes tratados também esteve aquém do esperado ${ }^{28}$.

Observamos na análise univariada que níveis baixos de ferritina foram relacionados com piora da funcionalidade, 
pontuação mais baixa no MoCA, na memória lógica I e dígitos ordem inversa (memória verbal e de trabalho e atenção). Enquanto o IST $<20 \%$ se relacionou com pontuação menor na fluência fonêmica (letra " $\mathrm{S}$ ") e na memória visual (TEPIC). Chaiben et al em um estudo brasileiro com 240 pacientes com DRC pré dialítica encontraram associação da alteração da função executiva com menores taxas de filtração glomerular ${ }^{29}$, porém, neste estudo, não foi avaliado metabolismo do ferro.

Tamura et al avaliaram 762 pacientes com DRC e vários níveis de TFGe para determinar a relação da anemia com declínio cognitivo e concluíram que anemia não foi independentemente associada com a função cognitiva basal e com o declínio cognitivo, importante ressaltar que os autores definiram como anemia um nível de hemoglobina sérica menor do que $13 \mathrm{~g} / \mathrm{dL}^{30}$. Esse achado não está em acordo com nossos dados visto que a hemoglobina esteve associada ao MoCA de forma independente, porém, neste mesmo modelo, a depressão também foi preditora dos níveis de função cognitiva. Além de ter havido uma tendência na escolaridade e na TFGe, sugerindo que o comprometimento cognitivo em pacientes com DRC pré dialítica é, provavelmente, multifatorial.

Em todos os modelos de regressão ajustados para variáveis sociodemográficas e àquelas associadas a hemoglobina e ao metabolismo do ferro, a depressão foi preditora independente de menores escores no MoCA em nosso estudo. Este dado é corroborado por uma importante 
revisão de Millan et al que avaliou as disfunções cognitivas em doenças psiquiátricas detalhando suas características e causas. Nesta revisão os autores detalham, entre outras, as ligações entre depressão e declínio cognitivo ${ }^{31}$.

Com relação a prevalência de outras alterações neuropsicológicas que podem interferir na cognição, a prevalência de Sonolência diurna (Epworth>10), AOS (Berlim alto risco) e Síndrome das pernas inquietas foi elevada, em acordo com o descrito na literatura ${ }^{8,32}$. Modelos experimentais mostram que o sistema monoaminérgico cerebral é suscetível neurotoxinas urêmicas e que esse sistema pode ser responsável pela alteração do padrão de sono comumente observado em pacientes com $\mathrm{DRC}^{5}$.

Avaliando as possíveis associações de dados clínicos e laboratoriais com testes neuropsicológicos observamos que, a despeito da alta prevalência de comorbidades, estas não impactaram significativamente na avaliação neuropsicológica. A HAS pode ter influenciado o percentual elevado de pacientes com características clínicas de AOS, pois uma das categorias do Questionário clínico de AOS de Berlin é a presença de hipertensão. Sintomas de pernas inquietas e sonolência diurna estiveram presentes em 35,2\% da amostra corroborando com a literatura ${ }^{9}$, porém sem relação com o IST e com o DM. Avaliamos se o DM poderia ser um fator confundidor devido a frequência aumentada de neuropatia periférica nesses pacientes, mas não houve relação (dados não mostrados). 
O estudo apresentou algumas limitações, sendo o tamanho da amostra a mais importante destas. As vantagens do presente estudo: foi feita triagem cognitiva ampla, incluindo avaliação da velocidade de processamento e funções executivas, e avaliação laboratorial extensa. Foram incluídos escalas e questionários de avaliação do sono e comorbidades associadas (AOS e Pernas Inquietas), e avaliação da depressão que muitas vezes não é considerada na avaliação clínica do paciente com DRC e interfere significativamente com a cognição.

\section{CONCLUSÃO}

Com relação a avaliação cognitiva, o nível sérico de hemoglobina foi associado com comprometimento cognitivo, assim como níveis de ferritina. Enquanto não encontramos associação com as demais variáveis associadas ao metabolismo do ferro em análise multivariada. A presença de depressão foi associada a menores escores no MoCA.

\section{AGRADECIMENTOS}

O presente trabalho foi realizado com apoio da Coordenação de Aperfeiçoamento de Pessoal de Nível Superior - Brasil (CAPES) - Código de Financiamento 001. A todos os pacientes e funcionários da Fundação IMEPEN. A Mary Hellen Teixeira Crivellari que contribuiu para a confecção do estudo e do artigo. 


\section{REFERÊNCIAS}

1. World Health Organization (WHO). Dementia; c2019 (acessado em 15 de janeiro de 2020). Disponível em: https://www.who.int/newsroom/fact-sheets/detail/dementia

2.Rodríguez JG, Gutierrez GG. Definition and prevalence of mild cognitive impairment. Rev Esp Geriatr Gerontol 2017;52(Suppl 1):3-6. http://dx.doi.org/10.1016/S0211-139x(18)30072-6

3.Sociedade Brasileira de Nefrologia. Censo Brasileiro de Diálise de 2018 (acessado em 15 de janeiro de 2020). Disponível em: https://www.sbn.org.br

4. Levey AS, Eckardt K-U, Tsukamoto Y, Levin A, Coresh J, Rossert $\mathrm{J}$, et al. Definition and classification of chronic kidney disease: a position statement from Kidney Disease: Improving Global Outcomes (KDIGO). Kidney Int 2005;67:2089-100.

http://dx.doi.org/10.1111/j.1523-1755.2005.00365.x

5.Viggiano D, Wagner CA, Martino G, Nedergaard M, Zoccali C, Unwin $\mathrm{R}$, et al. Mechanisms of cognitive dysfunction in CKD. Nat Rev Nephrol 2020;16:452-69. http://dx.doi.org/10.1038/s41581-020-0266-9

6. Paraizo MA, Almeida ALM, Pires LA, Abrita RSA, Crivellari MHT, Pereira BS, et al. Montreal Cognitive Assesment (MoCA) no rastreio de comprometimento cognitivo leve ( $\mathrm{CCL}$ ) em pacientes com doença renal crônica (DRC) pré-dialítica. J Bras Nefrol 2016;38:31-41. http://dx.doi.org/10.5935/0101-2800.20160006

7.Feng L, Yap KB, Ng TP. Depressive symptoms in older adults with chronic kidney disease: mortality, quality of life outcomes and correlates. Am J Geriatr Psychiatry 2013;21:570-9. http://dx.doi.org/10.1016/j.agp.2012.12.020

8. Ajayi SO, Adeoye AM, Raji YR, Tayo B, Salako BL, Ogunniyi A, et al. Self-reported Sleep Disorder and Ambulatory Blood Pressure Phenotypes in Patients with or without Chronic Kidney Disease: Findings from Ibadan CRECKID Study. West Afr J Med 2019;36:61-8. https://www.researchgate.net/publication/333173604 Self-

reported Sleep Disorder and_Ambulatory Blood Pressure Phenotyp es in Patients with or without Chronic Kidney Disease Findings fr om Ibadan CRECKID Study

9. Merlino G, Lorenzut S, Gigli GL, Romano G, Montanaro D, Moro A, et al. A Case-Control Study on Restless Legs Syndrome in Nondialyzed Patients with Chronic Renal Failure. Mov Disord 2010;25:1019-25. http://dx.doi.org/10.1002mds.23010

10. Ribeiro-Alves MA, Gordan PA. Diagnóstico de anemia em pacientes portadores de doença renal crônica. J Bras Nefrol 2014;36(1 Supl 1):911. http://dx.doi.org/10.5935/0101-2800.20145003

11. Murray-Kolb LE, Beard JL. Iron treatment normalizes cognitive functioning in young women. Am J Clin Nutr 2007;85:778-87. http://dx.doi.org/10.1093/ajen/85.3.778

12. Memória CM, Yassuda MS, Nakano EY, Forlenza OV. Brief screening for mild cognitive impairment: validation of the Brazilian version of the 
Montreal cognitive assessment. Int J Geriatr Psychiatry 2013;28:3440. http://dx.doi.org/10.1002/gps.3787

13.Shulman KI. Clock-drawing: is it the ideal cognitive screening test? Int ] Geriatr Psychiatry 2000;15:548-61.

http://dx.doi.org/10.1002/1099-1166(200006)15:6<548: :aidgps $242>3.0 . \mathrm{co} ; 2-\mathrm{u}$

14. Nitrini R, Lefèvre BH, Mathias SC, Caramelli P, Carrilho PEM, Sauaia $\mathrm{N}$, et al. Testes neuropsicológicos de aplicação simples para 0 diagnóstico de demência. Arq Neuropsiquiatr 1994;52:457-65. http://dx.doi.org/10.1590/S0004-282X1994000400001

15. Nitrini R, Caramelli P, Porto CS, Charchat-Fichman H, Formigoni AP, Carthery-Goulart MT, et al. Brief cognitive battery in the diagnosis of mild Alzheimer's disease in subjects with medium and high levels of education. Dement Neuropsychol 2007;1:32-6. http://dx.doi.org/10.1590/S1980-57642008DN10100006

16. Nitrini R, Caramelli P, Herrera JR. E, Charchat-Fichman H, Porto CS. Performance in Luria's fist-edge-palm test according to educational level. Cogn Behav Neurol 2005;18:211-4. http://dx.doi.org/10.1097/01.wnn.0000195292.48422.d5

17.Brucki SMD, Malheiros SMF,Okamoto IH, Bertolucci PHF. Dados normativos para o teste de fluência verbal categoria animais em nosso meio. Arq Neuropsiquiatr http://dx.doi.org/10.1590/S0004-282X1997000100009

18. Beato RG, Nitrini R, Formigoni AP, Caramelli P. Brazilian version of the Frontal Assessment Battery (FAB) Preliminary data on administration to healthy elderly. Dement Neuropsychol 2007;1:5965. http://dx.doi.org/10.1590/S1980-57642008DN10100010

19.Pfeffer RI, Kurosaki TT, Harrah CH Jr, Chance JM, Filos S. Measurement of functional activities in older adults in the community. J Gerontol 1982;37:323-9.

http://dx.doi.org/10.1093/geronj/37.3.323

20.Johns MW. A new method for measuring daytime sleepiness: the Epworth sleepiness scale. Sleep 1991;14:540-5. http://dx.doi.org/10.1093/sleep/14.6.540

21.Fröhlich AC, Eckeli AL, Bacelar A, Poyares D, Pachito DV, Stelzer $\mathrm{FG}$, et al. Brazilian consensus on guidelines for diagnosis and treatment for restless legs syndrome. Arq Neuropsiquiatr 2015;73:260-80. http://dx.doi.org/10.1590/0004-282X20140239

22. Beck AT, Ward CH, Mendelson M, Mock J, Erbaugh J. An inventory for measuring depression. Arch Gen Psychiatry 1961;4:561-71. http://dx.doi.org/10.1001/archpsyc. 1961.01710120031004

23.Amorim P. Mini International Neuropsychiatric Interview (MINI): validação de entrevista breve para diagnóstico de transtornos mentais. Rev Bras Psiquiatr 2000;22:106-15.

http://dx.doi.org/10.1590/S1516-4446000000300003

24.Lozoff B. Early Iron Deficiency Has Brain and Behavior Effects Consistent with Doapminergic Dysfunction. J Nutr 2011;141:740-6. http://dx.doi.org/10.3945/jn.110.131169 
25.INAF. Indicador de Analfabetismo Funcional. INAF BRASIL 2018 Resultados Preliminares (acessado em 16 de janeiro de 2020). Disponível em: http://acaoeducativa.org.br/wpcontent/uploads/2018/08/Inaf2018 Relat\%C3\%B3rio-ResultadosPreliminares v08Ago2018.pdf

26. Andrade-Oliveira V, Foresto-Neto O, Watanabe IKM, Zatz R, Câmara NOS. Inflammation in Renal Diseases: New and Old Players. Front Pharmacol 2019;10:1192.

http://dx.doi.org/10.3389/fphar.2019.01192

27.Instituto Brasileiro de Geografia e Estatística (IBGE) - PNAD - 2001 - 2009 - Taxa de analfabetismo Funcional (acessado em: 07/06/2015).

Disponível em:

http://seriesestatisticas.ibge.gov.br/series.aspx?t=taxa-

analfabetismo\&vcodigo $=$ PD384

28. Asaleh M, Videloup L, Lobbedez T, Lebreuilly J, Morello R, Thuillier Lecouf A. Improved Detection and Evaluation of Depression in Patients with Chronic Kidney Disease: Validity and Reliability of Screening (PHQ-2) and Diagnostic (BDI-FS-Fr) Tests of Depression in Chronic Kidney Disease. Kidney Dis 2019;5:228-38. http://dx.doi.org/10.1159/000497352

29. Chaiben VBO, Silveira TBD, Guedes MH, Fernandes JPA, Ferreira JHF, Beltrão J, et al. Cognition and renal function: findings from a Brazilian population. J Bras Nefrol 2019;41:200-7. http://dx.doi.org/10.1590/2175-8239-JBN-2018-0067

30.Tamura MJ, Vittinghoff E, Yang J, Go AS, Seliger SL, Kusek JW, et al. Anemia and risk for cognitive decline in chronic kidney disease. BMC Nephrol 2016;17:13. http://dx.doi.org/10.1186/s12882-016-0226-6 31. Millan MJ, Agid Y, Brune M, Bullmore ET, Carter CS, Clayton NS, et al. Cognitive dysfunctions in psychiatric disorders: characteristics, causes and the quest for improved therapy. Nature Reviews Drug Discovery 2012;11:141-68. http://dx. doi.org/10.1038/nrd3628

32. Hao G, Lu W, Huang J, Ding W, Wang P, Wang L, et al. Predialysis fluid overload linked with quality of sleep in patients undergoing hemodialysis. Sleep Med 2018;51:140-7.

http://dx.doi.org/10.1016/j.sleep.2018.07.011 